\title{
Contemporary Pattern of Pediatric Infective Endocarditis from Tertiary Care Centre of Northern India: A Single Centre Experience
}

\author{
Dinesh Kumar $^{1}$ Shikha Garg ${ }^{1}$ Dheeraj D. Bhatt ${ }^{1}$ \\ ${ }^{1}$ Department of Paediatric Cardiology, Division of Paediatric \\ Cardiology, Atal Bihari Vajpayee Institute of Medical Sciences and \\ Dr. Ram Manohar Lohia Hospital, New Delhi, India \\ J Child Sci 2021;11:e250-e254.
}

\begin{abstract}
Address for correspondence Dheeraj D. Bhatt, MD, DM, Department of Paediatric Cardiology, Division of Paediatric Cardiology, Atal Bihari Vajpayee Institute of Medical Sciences and Dr. Ram Manohar Lohia Hospital, New Delhi 110001, India

(e-mail: dheeraj491@hotmail.com).
\end{abstract}

\author{
Abstract \\ Keywords \\ - infective endocarditis \\ - pediatrics \\ - duke criteria \\ - blood culture- \\ negative infective \\ endocarditis
}

With an increasing number of children with congenital heart disease (CHD) undergoing corrective treatments, improved pediatric intensive care, better antimicrobial treatments, and a relative decrease in rheumatic heart disease over the years, the epidemiology of pediatric infective endocarditis in India may be undergoing a change. The study was done in the department of pediatrics of a tertiary care teaching hospital of North India. A retrospective analysis of case records of children ( $<12$ years) admitted with a diagnosis of infective endocarditis (IE) from January 2013 to April 2019 was performed. Modified Duke's criteria were used to diagnose IE. There were 21 children diagnosed with infective endocarditis during this period. The mean age at presentation was 70 months (range: $2.5-144$ months). CHD $(n=13 / 21,61.9 \%)$ was the most common predisposing condition. A total of $28 \%(6 / 21)$ patients had no preexisting structural heart disease. Nine percent $(2 / 21)$ had rheumatic heart disease. Staphylococcus aureus was the most common etiological agent in those with a structurally normal heart. Most patients had blood culture-negative infective endocarditis ( $n=12$, $57.1 \%)$. Only one patient fulfilled Duke's major microbiological criteria. Six patients (28.57\%) died during the hospital stay. Increasingly younger children are being diagnosed with infective endocarditis in India and a significant number of them are in the setting of a structurally normal heart. In view of high percentage of culturenegative endocarditis, the Duke criteria may need to be revised to retain their sensitivity in such settings.

\section{Introduction}

Infective endocarditis is an illness which still has a high rate of morbidity and mortality. ${ }^{1}$ Population and hospital-based data show an estimated incidence of 1.5 to 11.6 per 100,000 people in the general population. ${ }^{2}$ Compared with adults, infective endocarditis (IE) has a lower incidence and different epidemiological profile in children. Predisposing factors

received

April 6, 2021

accepted after revision

August 6, 2021
DOI https://doi.org/

10.1055/s-0041-1735881.

ISSN 2474-5871. often associated with IE in adults, such as intravenous drug abuse and degenerative heart disease, are not common in children. ${ }^{3-8}$ Children with congenital heart disease (CHD), especially those with cyanotic heart disease, are at an increased risk for developing infective endocarditis.

The American Heart Association (AHA) draft statement in 2015 acknowledges quoting a recent multicenter report ${ }^{9}$ that the annual incidence rate of IE in the United States

\section{(C) 2021. The Author(s).}

This is an open access article published by Thieme under the terms of the Creative Commons Attribution License, permitting unrestricted use, distribution, and reproduction so long as the original work is properly cited. (https://creativecommons.org/licenses/by/4.0/) Georg Thieme Verlag KG, Rüdigerstraße 14, 70469 Stuttgart, Germany 
was between approximately 0.05 and 0.12 cases per 1,000 pediatric admissions from 2003 to 2010. Also, it points toward an increasing overall frequency of endocarditis among children. It cites improved survival of at-risk children, such as those with CHD (with or without surgery) and hospitalized infants as a possible underlying cause.

There are differences in the epidemiological profile between the developed and developing countries. With the decline in the prevalence of rheumatic heart disease (RHD), IE has become relatively uncommon in this subset of patients in the developed world; in the past two decades, CHD has become the most important predisposing condition for IE in children aged 2 years and above. ${ }^{10}$

Improvement in health care has also contributed to the change in epidemiology of the disease in developing nations. On one hand, early surgical correction of CHD lesions (that were major risk factors for IE in the past) has changed the substrate for this disease. However, on the other hand, postoperative IE is a long-term risk after correction of complex CHD, especially in those with residual defects or in cases with previous corrective or palliative surgery, with or without implanted vascular grafts, patches, or prosthetic cardiac valves. $^{11-14}$

The situation is different in our country. Only a very small proportion of infants with CHD are fortunate enough to receive some form of palliation in India. ${ }^{15}$ Thus this epidemiological factor may not be applicable in the Indian setting. There is limited data on pediatric IE from India. Therefore, we did this study to evaluate the predisposing factors, microbiological profile, complications, and mortality in pediatric infective endocarditis among a Northern Indian population.

\section{Materials and Methods}

This study was done in the department of pediatrics of a tertiary care teaching hospital of North India (Atal Bihari Vajpayee Institute of Medical Sciences [ABVIMS] and Dr. Ram Manohar Lohia [RML] Hospital, New Delhi). Retrospective data of pediatric cases (age $<12$ years) diagnosed as infective endocarditis (fulfilling modified Duke's criteria) and admitted during January 2013 to April 2019 was collected from all available medical records and reviewed. Records were checked in the admission register of pediatric cardiology division, as well as pediatric cardiology clinic. Files of cases labeled as infective endocarditis were retrieved and details of cases were obtained, including medical history, examination findings, echocardiographic records, blood culture reports, treatment, and the course of the disease. All patients who were discharged were followed-up periodically in the pediatric cardiology clinic and their details were recorded. These records were also reviewed for follow-up data. Ethical clearance was taken from the institutional ethical committee.

In our center, patients suspected to be suffering from infected endocarditis were subjected to at least three blood cultures by separate venepunctures on the first day. If no growth was observed by the second day of incubation, another two to three blood cultures were sent. BACTEC was used as the culture method in our hospital. In severely ill and unstable patients, three separate venepunctures for blood cultures were performed over a short period of 1 to 2 hours and empirical antibiotic therapy was initiated. Follow-up blood cultures were obtained in those with positive blood cultures to document microbiological clearance or to diagnose persistent bacteremia

Echocardiography evaluation was performed by a pediatric cardiologist on a Philips 11HDXE station.

\section{Statistical Analysis}

Data were recorded in a predesigned proforma and managed on an Excel spreadsheet. The statistical analysis consisted of calculating mean, range, and proportions. The data were analyzed by using the SPSS Statistics software.

\section{Result}

Infective endocarditis (IE) was diagnosed in 21 patients out of 47,170 pediatric admissions. CHD (61.9\%), with a higher proportion of acyanotic heart disease, was the most common predisposing condition. However, six cases (28.5\%) had no underlying structural heart disease. None of them had a central venous catheter as a possible source of IE. ( - Table 1)

The median age at presentation was 78 months (range: 5-144 months) with male predominance (male-female ratio of 13:8). The average duration of symptoms before admission to the hospital was 1.25 months (range: 0.5-6 months). Fever (100\%, 21/21), splenomegaly (66.66\%, $14 / 21$ cases), and congestive heart failure $(61.9 \%, 13 / 21)$ were the major clinical features. Three patients had joint pain as one of the presenting symptoms but none of them had rheumatic heart disease and most likely had rheumatological manifestation of IE. Four

Table 1 Demographic data and underlying conditions children with infective endocarditis

\begin{tabular}{|l|l|}
\hline Variable & $\begin{array}{l}\text { Number (\%) } \\
n=21\end{array}$ \\
\hline Male & $13(61.9)$ \\
\hline $\begin{array}{l}\text { Median age (mo) }<5 \text { years } \\
\text { Age } \\
\text { Without prior structural } \\
\text { heart disease }\end{array}$ & $78(47.6)$ \\
\hline Rheumatic heart disease & $6(28.6)$ \\
\hline Congenital heart disease & $2(9.5)$ \\
\hline Ventricular septal defect & $13(61.9)$ \\
\hline $\begin{array}{l}\text { Ventricular septal defect and } \\
\text { atrial septal defect }\end{array}$ & $6(28.5)$ \\
\hline Patent ductus arteriosus & $1(4.75)$ \\
\hline Mitral valve prolapsed & $1(4.75)$ \\
\hline $\begin{array}{l}\text { The tetralogy of Fallot } \\
\text { (post-Blalock Taussig shunt) }\end{array}$ & $1(4.75)$ \\
\hline $\begin{array}{l}\text { The tetralogy of Fallot } \\
\text { (post-total correction) }\end{array}$ & $1(4.75)$ \\
\hline The tetralogy of Fallot (unoperated) & $2(9.5)$ \\
\hline
\end{tabular}


Table 2 Selected clinical and laboratory profile in children with infective endocarditis

\begin{tabular}{|l|l|}
\hline Variable & $\begin{array}{l}\text { Number (\%) } \\
\boldsymbol{n}=\mathbf{2 1}\end{array}$ \\
\hline Fever & $21(100)$ \\
\hline Splenomegaly & $14(66.6)$ \\
\hline Congestive heart failure & $13(61.9)$ \\
\hline Embolism & $9(42)$ \\
\hline Glomerulonephritis & $5(23.8)$ \\
\hline Rheumatoid factor positive & $5(23.8)$ \\
\hline Neurologic symptoms & $4(19)$ \\
\hline Joint symptoms & $3(14.2)$ \\
\hline Thigh abscess & $3(14.2)$ \\
\hline Roth's spots & $3(14.2)$ \\
\hline Janeway's lesions & $2(9.5)$ \\
\hline Dental caries & $2(9.5)$ \\
\hline
\end{tabular}

Table 3 Positive blood culture in children with infective endocarditis

\begin{tabular}{|l|l|}
\hline Microbial organism & $\begin{array}{l}\text { Different organisms } \\
\text { grown in blood } \\
\text { cultures (\%) } \\
\boldsymbol{n}=10\end{array}$ \\
\hline Staphylococcus aureus & $7(70)$ \\
\hline Streptococcus viridans & $2(20)$ \\
\hline Pseudomonas aeruginosa & $1(10)$ \\
\hline
\end{tabular}

children had neurological manifestations (one hemiparesis and three seizures; - Table 2). Twenty cases had acquired the infection from the community while one patient had developed endocarditis in the immediate postoperative period after undergoing total correction for the tetralogy of Fallot. Three patients had thigh abscess with two of them having underlying osteomyelitis.

Anemia (80\%, 17/21 cases), leukocytosis (33\%, 7/21), and microscopic hematuria $(23.8 \% n=5)$ were the important laboratory findings. Blood cultures were positive in nine patients (42.8\%; - Table 3 ).

The most common isolate was Staphylococcus aureus with all isolates being methicillin-sensitive (MSSA). One patient grew two organisms, Pseudomonas aeruginosa and $S$. aureus in separate cultures. This patient was admitted in the intensive care unit (ICU) with a short history. She presented with septic shock, skin gangrene, and vegetations on both aortic and mitral valves, and died before any surgical intervention could be done. She had a short hospital stay and grew $S$. aureus in one blood culture. The second blood culture grew $P$. aeruginosa; either organism could have been a true infection or a contaminant. All patients had received intravenous antibiotics before being admitted to our center. Only one patient fulfilled Duke's major clinical
Table 4 Frequency of patients fulfilling each of Duke's modified clinical criteria

\begin{tabular}{|l|l|}
\hline Criteria & Total (\%) \\
\hline Echo positive (major) & $21(100)$ \\
\hline Fever(minor) & $21(100)$ \\
\hline Predisposing condition (minor) & $15(71)$ \\
\hline Vascular (minor) & $12(57)$ \\
\hline Immunological (minor) & $11(52)$ \\
\hline Blood culture (minor) & $8(38)$ \\
\hline Blood culture (major) & $1(4.7)$ \\
\hline
\end{tabular}

criteria for blood culture with all the four blood cultures being positive for $S$. aureus.

Of the 12 blood culture-negative infective endocarditis (BCNIE) patients, 2 had thigh abscess, one of them grew methicillin-resistant while the other grew methicillin-sensitive $S$. aureus in pus culture. One patient had positive Widal's titers but the blood culture was sterile.

Using Duke's criteria as benchmark, almost all cases qualified as definite, with only one being labeled as possible infective endocarditis. Microbiological major criteria were least commonly fulfilled at presentation (-Table 4).

Vegetations were seen in all patients by transthoracic echocardiography. The mitral valve was most frequently involved followed by tricuspid and aortic valves. Multiple

Table 5 Echocardiographic features in children with infective endocarditis

\begin{tabular}{|c|c|}
\hline Vegetation & 21 \\
\hline \multicolumn{2}{|l|}{ Location } \\
\hline Isolated mitral & $7(33.3)$ \\
\hline Isolated tricuspid & $5(23.8)$ \\
\hline Isolated pulmonary & $2(9.5)$ \\
\hline Aortic and mitral & $2(9.5)$ \\
\hline Tricuspid and pulmonary & $1(4.7)$ \\
\hline Isolated aortic & $1(4.7)$ \\
\hline $\begin{array}{l}\text { Others (Patent ductus arteriosus, } \\
\text { Blalock Taussig shunt, ventricular } \\
\text { septal defect prosthetic patch) }\end{array}$ & $3(14.28)$ \\
\hline \multicolumn{2}{|l|}{ Size } \\
\hline$<10 \mathrm{~mm}$ & $1(4.7)$ \\
\hline$>10 \mathrm{~mm}$ & $20(95.2)$ \\
\hline \multicolumn{2}{|l|}{ Number } \\
\hline Single & $16(76.2)$ \\
\hline Multiple & $5(23.8)$ \\
\hline \multicolumn{2}{|l|}{ Mechanical complications } \\
\hline Abscess & $1(4.7)$ \\
\hline Chordal rupture & $1(4.7)$ \\
\hline
\end{tabular}


vegetations were seen in $23.8 \%$ with most of them greater than $1 \mathrm{~cm}$ (-Table 5 ).

Congestive heart failure and embolic features were seen in 13 (61.9\%) and 9 (42\%) patients, respectively.

Antibiotic protocols were as per AHA update recommendations on infective endocarditis in childhood. Three patients underwent surgery as a part of treatment: two survived and the third died in the hospital due to congestive heart failure.

Six patients (28.57\%) died during the hospital stay. The causes of mortality were sepsis, congestive heart failure and cardiogenic shock. Two patients died after discharge from the hospital. One patient was with a known septic aneurysm in the pulmonary artery and had sudden death at home after remaining afebrile for 2 months. Another child with a ventricular septal defect and vegetations in tricuspid valve remained afebrile and well till 6 months of posttreatment, after which she had a bout of hemoptysis followed by death.

\section{Discussion}

In our study, the mean age of the patients was 5.8 years (range: 2.5 months-12 years) with $19 \%(n=4)$ of the patients being younger than 2 years of age. This age group is younger compared with previous series on pediatric IE published from India. CHD ( $n=13 / 21,61 \%)$ was the major predisposing condition in pediatric IE in our cohort. Before the 1970s, 30 to $50 \%$ of U.S. children with IE had underlying rheumatic heart disease. In the past two decades, CHD has become the predominant underlying condition for IE in children from the developed world who are $>2$ years of age. ${ }^{10}$ Adult studies from India still report rheumatic heart disease as the main predisposing condition. ${ }^{16}$

In our study, 28.5\% (6/21) patients with IE had no preexisting structural heart disease which is significantly higher than that reported in the Western literature, wherein approximately 8 to $10 \%$ of pediatric IE develops without structural heart disease. ${ }^{13}$ Poor socioeconomic conditions, malnutrition, poor sanitation, overcrowded living, illiteracy, lack of health care, and immunization may account for increased vulnerability to severe infections in infancy and childhood. In these patients, $S$. aureus was the causative organism in five out of six patients (three cases with blood culture and two with pus culture positive for $S$. aureus). No patient had an indwelling catheter, prematurity or chronic renal disease as a risk factor for infective endocarditis. A previous Indian study has reported that $16.6 \%(n=6 / 37)$ of IE patients had structurally normal heart with $S$. aureus being the commonest causative organism. ${ }^{5}$

Notably, a prospective study has found the incidence of infective endocarditis in children with staphylococcal bacteremia to be up to $20 \%{ }^{17}$ It appears that staphylococcal infection is itself a predisposing factor for infective endocarditis. Thus, pediatricians treating children with staphylococcal infections need to keep a high index of suspicion for infective endocarditis. Conversely one should actively look for extracardiac foci of infection, for example, pustules, osteomyelitis, septic arthritis, and others, in children with staphylococcal IE.
Most of our patients had BCNIE $(n=12,57.1 \%)$. However, in the West, it usually represents 2.5 to $31 \%$ of infective endocarditis. ${ }^{11}$ Since all the enrolled patients in our study had received antibiotics before admission, the high culture negativity was to be expected and is consistent with other publications from India., 4,7,8

Echocardiography findings and fever were present in all of our patients. Blood culture positivity was seen in $42.8 \%$.This is similar to previous reported pediatric studies from India. Only one patient fulfilled Duke's major microbiological criteria. The Duke criteria have been previously validated in children. ${ }^{18,19}$ However, the reported sensitivity and specificity of Duke's criteria in a recent study from Cleveland clinic was only around $70 \%{ }^{20}$ The sensitivity of Duke's clinical criteria decreased further in the presence of culture-negative endocarditis. Lamas and Eykyn have reported a sensitivity of $21 \%$ in bacterial culture-negative endocarditis. ${ }^{21}$ Since predisposing cardiac conditions is a minor criterion for IE, the increasing occurrence of infective endocarditis in a normal heart will likely further decrease the sensitivity of Duke's criteria. Easy availability of over the counter antibiotics and high rate of prior antibiotic use (such as prevalent in our setting), inevitably leads to high rates of blood culture negativity and difficulty in fulfilling Duke's microbiological criteria. In the context of frequent prior antibiotic use and detection of vegetation on echocardiography, a case can be made for considering even a single positive blood culture (especially for S. aureus) as a major criterion. Role of other imaging like fluorodeoxyglucose (FDG) positron emission tomography (FDG PET) should be evaluated in BCNIE and possibly incorporated in the modified Duke criteria. Since pediatric IE has such a low incidence, multicenter, prospective collaborative studies are required to collect data on this subject as was done in a recent publication based on adult patients with IE. ${ }^{1}$

Rheumatological manifestations occur in 25 to $42 \%$ of adults with infective endocarditis. This phenomenon has not been studied well in pediatric cases. ${ }^{22}$ In our cohort, three patients had joint pain as one of the presenting symptoms of infective endocarditis; none of them had rheumatic heart disease. Fever with joint involvement should prompt early consideration of infective endocarditis as one of the differential diagnosis. Treating physicians need to be aware that rheumatological manifestations also fall within the spectrum of manifestations of IE.

Our in-hospital mortality (30\%) is comparable to that reported from other studies (25\% Bhat et al, ${ }^{6} 43 \%$ Dhawan et $\mathrm{al}^{5}$ and $26 \%$ Saxena et $\mathrm{al}^{8}$ ) from India but substantially higher than that reported in the West (5\% Day et al ${ }^{11}$ and $2.8 \%$ Gupta et al, ${ }^{13}$ both from the United States). ${ }^{1,9,11}$ Death due to complications, like mycotic pulmonary aneurysms, in the follow-up period underscores the importance of frequent follow-up and a careful and detailed assessment on each follow-up visit.

\section{Limitations}

The major limitations of our study were the small number of patients, retrospective data analysis, and a lack of 
pathological confirmation of the diagnosis of infective endocarditis. Collection of weight-based standardized volumes of blood could not be confirmed from the records, as this was a retrospective study. Also, the results of the study cannot be generalized as it was a single-center hospital-based study. The strength of the study was that all but one patient had definite infective endocarditis.

\section{Conclusion}

Increasingly younger children are being diagnosed with infective endocarditis in India and a significant number of them are in the setting of a structurally normal heart. There is high in-hospital mortality in these patients. S. aureus continues to be the main organism causing IE. There is of high proportion of BCNIE presumably because of prior antibiotic use. Even in blood culture-positive cases, most of the patients only fulfilled Duke's minor microbiological criteria (blood culture). As this is a rare disease, multicenter studies may be needed to study a larger number of patients. Also, Duke's microbiological criteria may need to be revisited, so as to not miss out on the diagnosis of this potentially life-threatening disease. There is a need for increased awareness of the spectrum of disease manifestations, and close postdischarge follow-up among pediatricians and pediatric cardiologists.

\section{Ethical Approval}

Approval of the institutional ethical committee was obtained for this study.

\section{Funding}

None.

\section{Conflict of Interest}

None declared.

\section{References}

1 Habib G, Erba PA, Iung B, et al; EURO-ENDO Investigators. Clinical presentation, aetiology and outcome of infective endocarditis. Results of the ESC-EORP EURO-ENDO (European infective endocarditis) registry: a prospective cohort study. Eur Heart J 2019;40 (39):3222-3232

2 Bin Abdulhak AA, Baddour LM, Erwin PJ, et al. Global and regional burden of infective endocarditis, 1990-2010: a systematic review of the literature. Glob Heart 2014;9(01):131-143

3 Kohli RS, Anand IS, Nagrani B, et al. Infective endocarditis in children. Indian Pediatr 1983;20(06):439-443

4 Bhandari S, Kaul U, Shrivastava S, Tandon R, Bhatia ML. Infective endocarditis in children. Indian J Pediatr 1984;51(412):529-532
5 Dhawan A, Grover A, Marwaha RK, et al. Infective endocarditis in children: profile in a developing country. Ann Trop Paediatr 1993; 13(02):189-194

6 Bhat AW, Jalal S, John V, Bhat AM. Infective endocarditis in infants and children. Indian J Pediatr 1996;63(02):204-209

7 Sharma M, Saxena A, Kothari SS, et al. Infectious endocarditis in children: changing pattern in a developing country. Cardiol Young 1997;7(02):201-206

8 Saxena A, Aggarwal N, Gupta P, Juneja R, Kothari SS, Math R. Predictors of embolic events in pediatric infective endocarditis. Indian Heart J 2011;63(03):237-240

9 Pasquali SK, He X, Mohamad Z, et al. Trends in endocarditis hospitalizations at US children's hospitals: impact of the 2007 American Heart Association Antibiotic Prophylaxis Guidelines. Am Heart J 2012;163(05):894-899

10 Elder RW, Baltimore RS. The changing epidemiology of pediatric endocarditis. Infect Dis Clin North Am 2015;29(03):513-524

11 Day MD, Gauvreau K, Shulman S, Newburger JW. Characteristics of children hospitalized with infective endocarditis. Circulation 2009;119(06):865-870

12 Esposito S, Mayer A, Krzysztofiak A, et al; Italian Pediatric Infective Endocarditis Registry. Infective endocarditis in children in Italy from 2000 to 2015. Expert Rev Anti Infect Ther 2016;14(03): 353-358

13 Gupta S, Sakhuja A, McGrath E, Asmar B. Trends, microbiology, and outcomes of infective endocarditis in children during 20002010 in the United States. Congenit Heart Dis 2017;12(02): 196-201

14 Rosenthal LB, Feja KN, Levasseur SM, Alba LR, Gersony W, Saiman L. The changing epidemiology of pediatric endocarditis at a children's hospital over seven decades. Pediatr Cardiol 2010;31 (06):813-820

15 Saxena A. Congenital heart disease in India: a status report. Indian Pediatr 2018;55(12):1075-1082

16 Senthilkumar S, Menon T, Subramanian G. Epidemiology of infective endocarditis in Chennai, South India. Indian J Med Sci 2010;64(04):187-191

17 Valente AM, Jain R, Scheurer M, et al. Frequency of infective endocarditis among infants and children with Staphylococcus aureus bacteremia. Pediatrics 2005;115(01):e15-e19

18 Stockheim JA, Chadwick EG, Kessler S, et al. Are the Duke criteria superior to the Beth Israel criteria for the diagnosis of infective endocarditis in children? Clin Infect Dis 1998;27(06):1451-1456

19 Tissières P, Gervaix A, Beghetti M, Jaeggi ET. Value and limitations of the von Reyn, Duke, and modified Duke criteria for the diagnosis of infective endocarditis in children. Pediatrics 2003; 112(6 pt 1):e467

20 Shrestha N, Shakya S, Hussain S, Pettersson G, Griffin B, Gordon S. Sensitivity and specificity of duke criteria for diagnosis of definite infective endocarditis: a cohort study. Open Forum Infect Dis 2017;4(01):550-551

21 Lamas CC, Eykyn SJ. Blood culture negative endocarditis: analysis of 63 cases presenting over 25 years. Heart 2003;89(03):258-262

22 González-Juanatey C, González-Gay MA, Llorca J, et al. Rheumatic manifestations of infective endocarditis in non-addicts. A 12-year study. Medicine (Baltimore) 2001;80(01):9-19 